\title{
Psychoacoustic Approach to Machine Fault Diagnosis
}

\author{
Primož Potočnik, Edvard Govekar, Igor Grabec and Peter Mužič \\ University of Ljubljana, Faculty of Mechanical Engineering, Aškerčeva 6, SI-1000 Ljubljana, Slovenia
}

(Received 16 June 2005; accepted 10 August 2005)

\begin{abstract}
A method for machine diagnosis, based on acoustic signals, is proposed. The method is based on psychoacoustic modelling, which simulates the ability of human aural analysis. The solution approach combines psychoacoustic pre-processing, feature-extraction modules, and feature-evaluation modules. Feature-extraction methods are defined for the detection of stationary and dynamic acoustic phenomena. Stationary features are appropriate for diagnosing machines that emit continuous sounds, and defects are reflected in altered sound patterns. Dynamic features are suitable for the detection of transient phenomena, such as collisions, etc. Statistical procedures, based on asymmetrically defined fault-detection margins, are proposed for an evaluation of extracted features. An application of the method for the diagnosis of industrially produced compressors is discussed. Noxious space defect and lubrication defect are diagnosed by a stationary approach during the normal operation of compressors. Various mechanical defects in the supporting spring system are diagnosed by extracting dynamic features for collision detection. Compressor defects are successfully recognised through the proposed method.
\end{abstract}

\section{INTRODUCTION}

Modern industrial production, based on machining processes, requires reliable condition-monitoring procedures. Through the proper monitoring of machines, a harmful machine breakdown and, consequently, a loss of production can be prevented. Various sensory signals - such as acoustic emission, temperature, vibration, and forces - can be monitored in order to estimate the machine's condition. ${ }^{1,2}$ Machinery fault diagnosis, based on vibroacoustic signals, is currently receiving considerable attention. ${ }^{3-5}$ Components of rotating machinery that suffer from wear can be diagnosed through the appropriate methods. ${ }^{6-8}$

Machine-structure-borne sound is also very informative for condition monitoring and fault diagnosis. Human operators can often diagnose machine defects while the machine is in operation, by simply performing an aural inspection. Such a method requires a clear distinction between sound patterns of normal and abnormal machine operation. Various successful applications of acoustic-based machine fault detection are reported in the literature. Tsao et al. applied a neural network to acoustic signatures of induction motors in order to recognise motor faults. ${ }^{9} \mathrm{Li}$ et al. proposed a neural-network-based expert system for machine fault diagnosis in which acoustic power spectra and statistical moments were used as input features. ${ }^{10}$ Sound analysis with an extraction of features for particular defect types was proposed by Benko et al. and the method was applied to a vacuum cleaner fault diagnosis. ${ }^{11}$ Lin applied a Morelet wavelet, for the extraction of the features of machine sounds for the purposes of machine fault diagnosis. ${ }^{12}$ An application of a hidden Markov model, which is a successful method for speech recognition, was proposed for the diagnosis of mechanical faults. ${ }^{13}$ The idea of an electronic stethoscope was proposed by Tse et al., in which a direct analysis of machine sound for machine fault diagnosis is based on continuous wavelet transformation and on trajectory parallel measures. ${ }^{14}$
In this paper, we propose a method for automatic acousticbased machine diagnosis that resembles an artificial ear. The method is based on psychoacoustic modelling, which describes the ability of human aural analysis. ${ }^{15}$ Psychoacoustic models combine several signal-processing stages for simulating aspects of transformation in the auditory periphery. ${ }^{16} \mathrm{~A}$ pre-processing stage combines an array of independent bandpass filters with signal rectification and with low-pass filtration. This stage roughly simulates the transformation of mechanical oscillations of the basilar membrane into receptor potentials in the inner hair cells. Further stages combine nonlinear adaptation for compression and high sensitivity for fast temporal variations. Finally, signals are analysed by a decision device.

The proposed method combines psychoacoustic modelling with simple signal-processing routines. The method is suitable for detection of both stationary and dynamic acoustic phenomena. For the former, faults can be detected in machines that emit continuous sounds, - defects occur only as slight alterations from a basic buzz pattern. For the latter, dynamic acoustic phenomena, such as transients caused by collisions and other events, can be detected.

This article is organised as follows: a solution approach is introduced first, which is followed by a description of psychoacoustic pre-processing. The next section presents feature-extraction methods for detecting stationary and dynamic acoustic phenomena. Extracted features are then evaluated by statistical procedures that diagnose a normal or faulty state. A section with industrial applications presents two case studies that illustrate stationary and dynamic sound recognition. Both case studies address the production of commercial compressors and focus on the recognition of various defect types.

\section{SOLUTION APPROACH}

The proposed method of automatic aural detection of machining phenomena consists of three successive modules which are schematically shown in Fig. 1. 